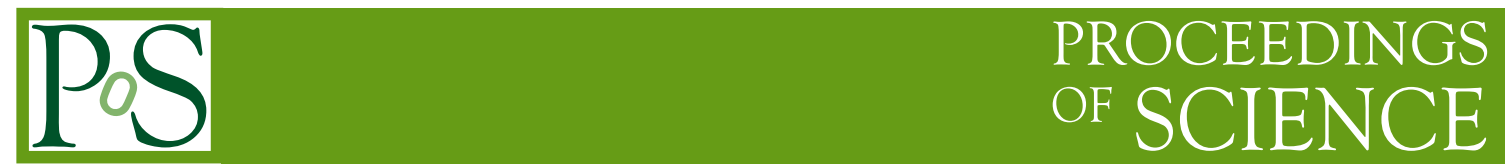

\title{
Charmed pentaquark searches at HERA
}

\author{
Dmitri Ozerov*t \\ ITEP Russia / DESY Germany \\ E-mail: ozerov@mail.desy.de
}

Recent results from HERA experiments on searches of an exotic baryon decaying into $D^{*} p$ are summarised. The observation by the H1 Collaboration of a resonance at the mass $3099 \pm 3($ stat $) \pm$ 5 (syst) $\mathrm{MeV}$ with narrow width of $12 \pm 3$ (stat) $\mathrm{MeV}$ is discussed and details in form of inclusive and differential production cross sections and acceptance corrected ratio $\sigma\left(D^{*} p(3100)\right) / \sigma\left(D^{*}\right)$ are presented. A search with negative result, made by the ZEUS Collaboration of such a resonance in their data is reviewed.

International Europhysics Conference on High Energy Physics July 21st - 27th 2005

Lisboa, Portugal

\footnotetext{
* Speaker.

$\dagger$ on behalf of the $\mathrm{H} 1$ and ZEUS Collaborations
} 


\section{Introduction}

In the past years experimental evidences[1] of a exotic baryon state with strangeness +1 brought new excitation in the field of hadron spectroscopy. The amazing coincidence of the experimental results on both the mass of the state and the unique narrow width with the theoretical prediction[2] (made several years before the first experimental evidence) stimulated experimentalists and theoreticians to look deeper into the subject. Right now the situation concerning the existence of the strange pentaquark is completely unclear from both the experimental (because of the large number of experiments where the pentaquark was not observed[1]) and theoretical points of view. Nevertheless efforts to find such an object and to describe it have already contributed significantly to hadron physics by popularising it and giving new ideas and methods.

The probable existence of a strange pentaquark with quark content $u u d d \bar{s}$ implies that a charmed pentaquark with the content $u u d d \bar{c}$ could also exist. If the mass of such a state is above the sum of the masses of $D^{*}$ and proton, it may decay to $D^{* \pm} p^{\mp}$. In the current paper the searches of the charmed pentaquark made by the $\mathrm{H} 1$ and ZEUS Collaborations at HERA are reviewed.

\section{Observation of a $D^{*} p$ resonance}

The $\mathrm{H} 1$ Collaboration found [3] a narrow signal in the $D^{* \pm} p^{\mp}$ invariant mass at $3.1 \mathrm{GeV}$ with a width consistent with the detector resolution. The $D^{*}$ mesons were identified using the $D^{* \pm} \rightarrow D^{0} \pi^{ \pm} \rightarrow\left(K^{\mp} \pi^{ \pm}\right) \pi^{ \pm}$decay channel. A clean signal is seen in DIS $\left(Q^{2}>1.0 \mathrm{GeV}^{2}\right)$ and, independently, in the photoproduction sample $\left(Q^{2}<1.0 \mathrm{GeV}^{2}\right)$, as shown in figure 1. The probability of the background to fluctuate to the signal in the DIS sample was estimated to be less than $4 \cdot 10^{-8}$ which corresponds to $5.4 \sigma$ in terms of Gaussian standard deviations.
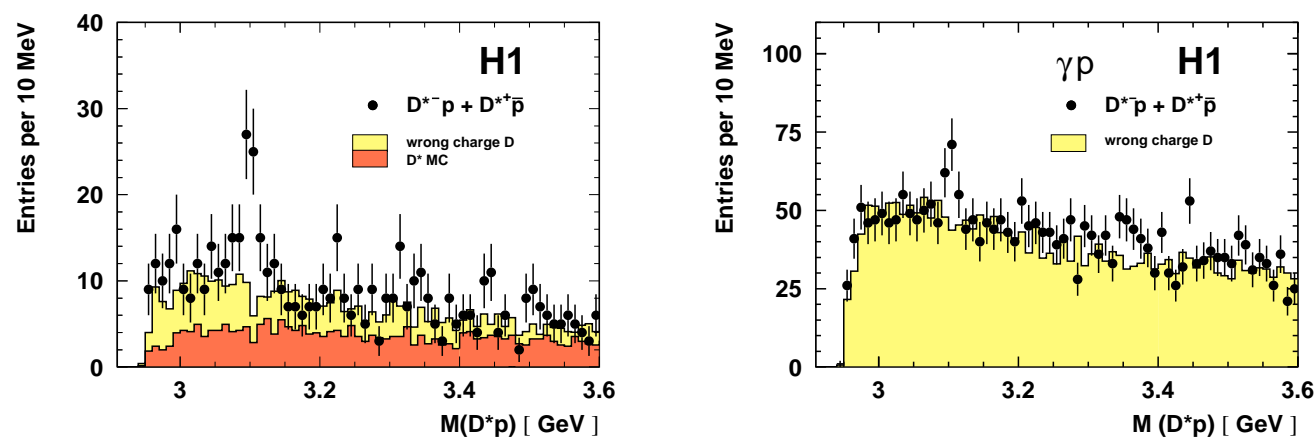

Figure 1: Invariant mass of opposite sign $D^{*} p$ combinations for DIS (left) and photoproduction (right) data samples of the H1 Collaboration.

\subsection{Analysis of $D^{*} p$ combinations}

A total acceptance corrected yields ratio $R_{c o r}\left(D^{*} p(3100) / D^{*}\right)=\left(1.59 \pm 0.33(\text { stat })_{-0.45}^{+0.33}(\right.$ syst $\left.)\right) \%$, has been measured in the visible range of the $D^{*} p(3100): p_{t}>1.5 \mathrm{GeV},-1.5<\eta<1.0$ and of the $D^{*}$ meson: $p_{t}>1.5 \mathrm{GeV},-1.5<\eta<1.0, z>0.2$. If further corrections to the $D^{*} p(3100)$ signal are applied by extrapolating to the full $D^{*}$ phase space, the visible cross section ratio is 

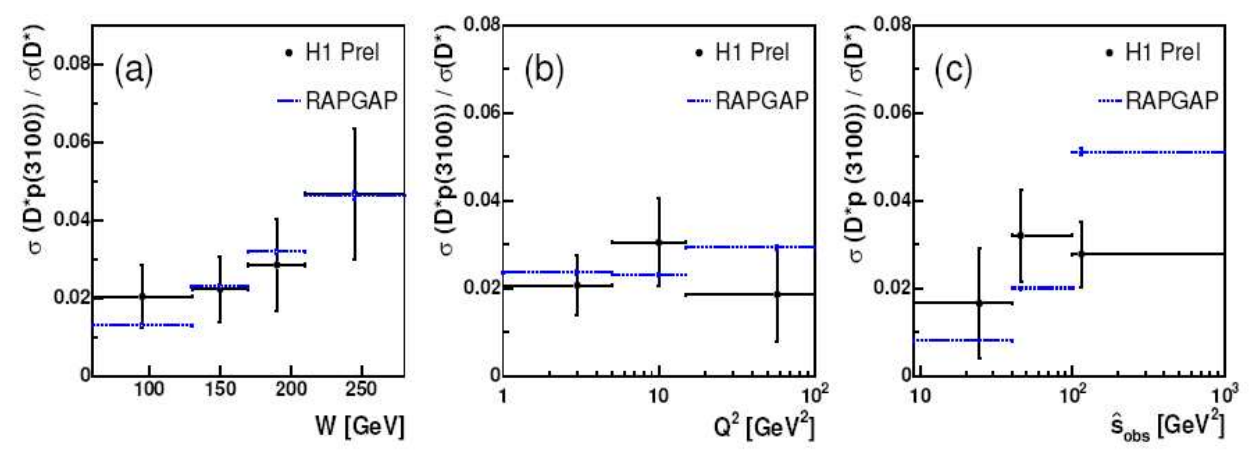

Figure 2: $\sigma_{v i s}\left(D^{*} p(3100)\right) / \sigma_{v i s}\left(D^{*}\right)$ as a function of the kinematic variables (a) $\mathrm{W}$, (b) $Q^{2}$ and (c) $\hat{s}_{o b s}$. Data (closed symbols) are compared with the expectation (dashed line) of RAPGAP 3.1 which assumes the same mechanism for $D^{*}$ and $D^{*} p(3100)$ production. Only statistical errors are shown.

$\sigma_{v i s}\left(D^{*} p(3100) / \sigma_{v i s}\left(D^{*}\right)=\left(2.48 \pm 0.52(\text { stat })_{-0.64}^{+0.85}\right) \%\right.$. In figure 2 the acceptance corrected ratio $\sigma_{v i s}\left(D^{*} p(3100)\right) / \sigma_{v i s}\left(D^{*}\right)$ is shown as a function of the proton photon centre-of-mass energy $W$, the four momentum transfer squared of the virtual photon $Q^{2}$ and the invariant mass of the $c \bar{c}$ system $\hat{s}_{o b s}$, in comparison with the expectations of the fragmentation production model, which assumes the same production mechanism of the $D^{*} p(3100)$ as for the $D^{*}$ mesons and isotropical decay to $D^{*}$ and proton. Since the absolute normalization of the $D^{*} p(3100)$ rate is not fixed in the model, the $D^{*} p(3100)$ yield is normalized such that the ratio $R_{c o r}$ is reproduced. The observed dependence on $\mathrm{W}$ and $Q^{2}$ is well described by this model, while it is significantly above the data at large $\hat{s}_{\text {obs }}$.
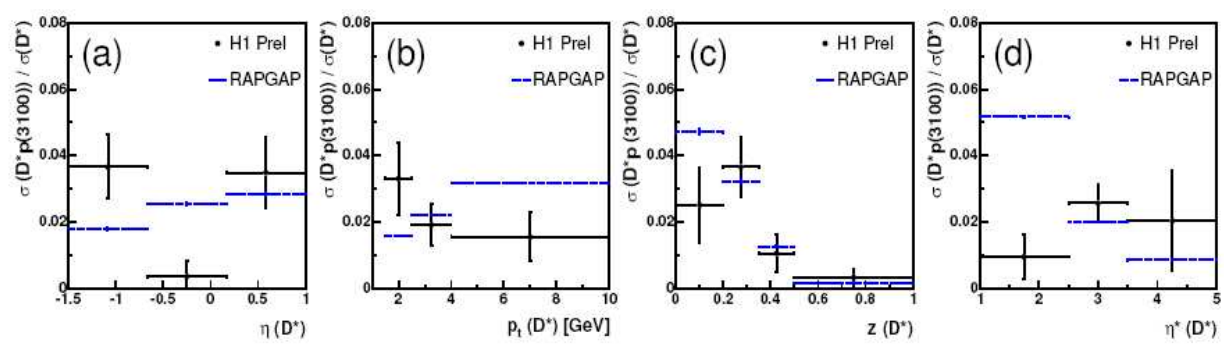

Figure 3: $\sigma_{v i s}\left(D^{*} p(3100)\right) / \sigma_{v i s}\left(D^{*}\right)$ as a function of the $D^{*}$ variables (a) $\eta\left(D^{*}\right)$ and (b) $p_{t}\left(D^{*}\right)$ both in the laboratory frame, (c) $z\left(D^{*}\right)$ and (d) $\eta^{*}\left(D^{*}\right)$ in the hadronic centre-of-mass system.

In order to investigate the kinematics of the $D^{*}$ mesons contributing to the $D^{*} p(3100)$ resonance the ratio $\sigma_{v i s}\left(D^{*} p(3100)\right) / \sigma_{v i s}\left(D^{*}\right)$ is shown in figure 3 as a function of the pseudorapidity $\eta\left(D^{*}\right)$ and the transverse momentum $p_{t}\left(D^{*}\right)$, both in the laboratory frame, the inelasticity $z\left(D^{*}\right)$ and the pseudorapidity $\eta^{*}\left(D^{*}\right)$ in the hadronic centre-of-mass system. Also shown are the expectations from the model. The most striking feature in the data is the suppression of the $D^{*} p(3100)$ baryon relative to $D^{*}$ meson production in the central region in both frames. Such a dependence is not predicted by the fragmentation production model. The data indicate that the $D^{*} p(3100)$ baryon 
production is closer to the photon direction than normal $D^{*}$ meson production.
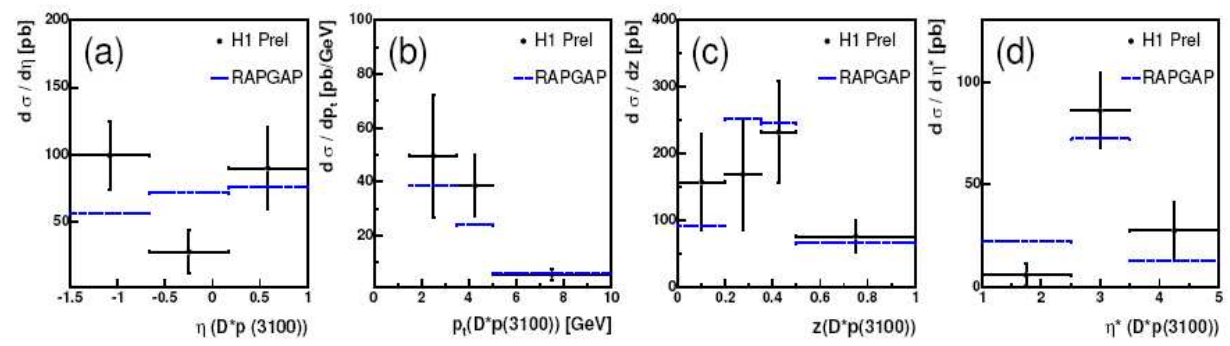

Figure 4: Differential $D^{*} p(3100)$ cross sections as a function of $D^{*} p$ variables (a) $\eta\left(D^{*} p\right)$ and (b) $p_{t}\left(D^{*} p\right)$, (c) $z\left(D^{*} p\right)$ and (d) $\eta^{*}\left(D^{*} p\right)$.

In figure 4 the $D^{*} p(3100)$ differential cross sections are presented as a function of $\eta\left(D^{*} p\right)$, $p_{t}\left(D^{*} p\right), z\left(D^{*} p\right)$ and $\eta^{*}\left(D^{*} p\right)$. The $D^{*} p(3100)$ production cross section shows the same features as a function of $\eta\left(D^{*} p\right)$ and $\eta^{*}\left(D^{*} p\right)$ as observed for the ratio $\sigma_{v i s}\left(D^{*} p(3100)\right) / \sigma_{v i s}\left(D^{*}\right)$ as a function of the corresponding $D^{*}$ variables. Within the quite large statistical errors the shapes of the $z\left(D^{*} p\right)$ and of the $p_{t}\left(D^{*} p\right)$ distributions are consistent with the fragmentation production model. These two distributions are suggesting that boson gluon fusion is the source of production of $D^{*} p(3100)$ baryons while the pseudorapidity distributions are not evidently supporting this picture.
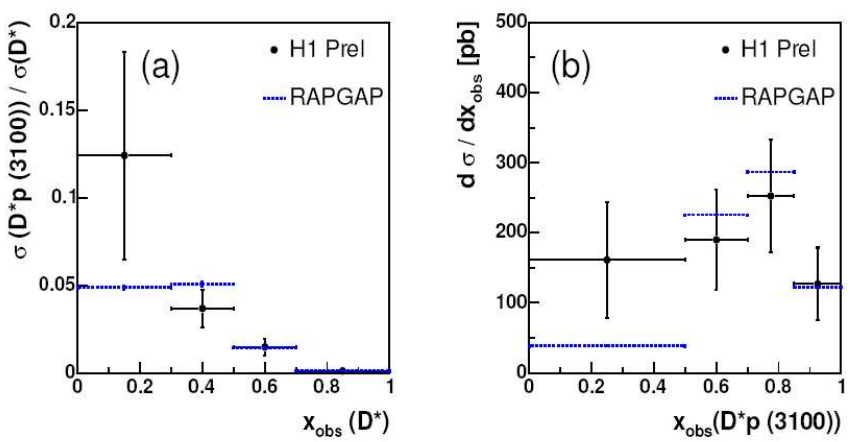

Figure 5: $\sigma_{v i s}\left(D^{*} p(3100)\right) / \sigma_{v i s}\left(D^{*}\right)$ as a function of the $D^{*}$ hadronization fraction $x_{o b s}\left(D^{*}\right)$ in (a) and $d \sigma_{v i s}\left(D^{*} p(3100)\right) / d x_{o b s}\left(D^{*} p\right)$ as a function of the $x_{o b s}\left(D^{*} p\right)$ in (b).

Finally, information on the $D^{*} p(3100)$ fragmentation process and the $D^{*}$ hadronization of $D^{*}$ mesons from $D^{*} p(3100)$ decay has been extracted from the data. In figure 5 the cross section ratio $\sigma_{v i s}\left(D^{*} p(3100)\right) / \sigma_{v i s}\left(D^{*}\right)$ as a function of the $D^{*}$ hadronization variable $x_{o b s}\left(D^{*}\right)$ (the fraction of the energy of the charm quark taken by the $D^{*}$ meson) and the differential $D^{*} p(3100)$ cross section as a function of the fragmentation variable $x_{o b s}\left(D^{*} p\right)$ are shown together with the predictions from the model. The ratio $\sigma_{v i s}\left(D^{*} p(3100)\right) / \sigma_{v i s}\left(D^{*}\right)$ (figure 5a) increases with decreasing $x_{o b s}\left(D^{*}\right)$ value, indicating that $D^{*}$ mesons originating from $D^{*} p(3100)$ decays are significantly softer than inclusive $D^{*}$ mesons. This is expected in the decay of a real $D^{*} p(3100)$ particle. In figure $5 \mathrm{~b}$ the differential cross section $d \sigma_{v i s}\left(D^{*} p(3100)\right) / d x_{\text {obs }}\left(D^{*} p\right)$ is shown as a function of $x_{o b s}\left(D^{*} p\right)$. 


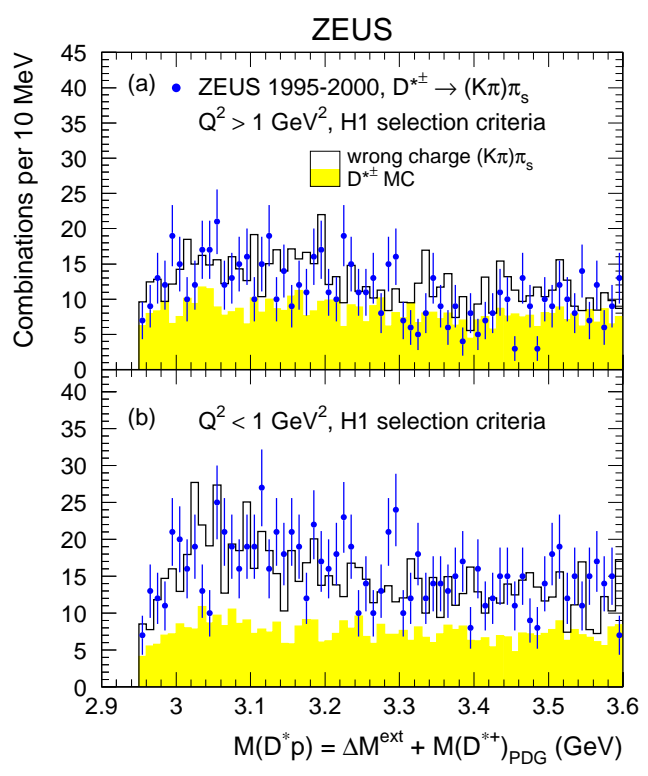

Figure 6: Invariant mass of the opposite signs $D^{*} p$ combinations for the DIS (a) and photoproduction (b) data samples of the ZEUS Collaboration with selection criteria similar to that of the H1 Collaboration.

The $D^{*} p(3100)$ fragmentation function(figure $5 \mathrm{~b}$ ) appears hard compared to the $D^{*}$ hadronization function. Such hard fragmentation is expected for charmed hadrons.

\section{Search by the ZEUS Collaboration}

The ZEUS Collaboration made a similar search for such a state [4]. The ZEUS $D^{*}$ mesons were reconstructed using two decay channels of the $D^{0}, D^{0} \rightarrow K^{\mp} \pi^{ \pm}$and $D^{0} \rightarrow K^{\mp} \pi^{ \pm} \pi^{+} \pi^{-}$. The integrated luminosity of the ZEUS data sample is 1.7 times larger than that of the H1 data sample. No narrow signal was observed in neither of the $D^{0}$ decay modes. The upper limits on the $R_{\text {cor }}\left(D^{*} p(3100) / D^{*}\right)$ are $<0.59 \%$ for the $K^{\mp} \pi^{ \pm}$decay channel and $<0.51 \%$ for both channels. The selection criteria were different in the ZEUS analysis compared to the H1 measurement, resulting in different phase space regions explored and in a larger yield of reconstructed $D^{*}$ in the ZEUS investigation. The ZEUS Collaboration also presented results in a phase space region similar to the H1 study and found no indication of a narrow resonance, neither in the DIS nor in the photoproduction sample (see figure 6).

\section{References}

[1] K. H. Hicks, Prog. Part. Nucl. Phys. 55 (2005) 647.

[2] D. Diakonov, V. Petrov and M. V. Polyakov, Z. Phys. A 359 (1997) 305.

[3] A. Aktas et al. [H1 Coll.], Phys. Lett. B 588 (2004) 17.

[4] S. Chekanov et al. [ZEUS Coll.], Eur. Phys. J. C 38 (2004) 29. 